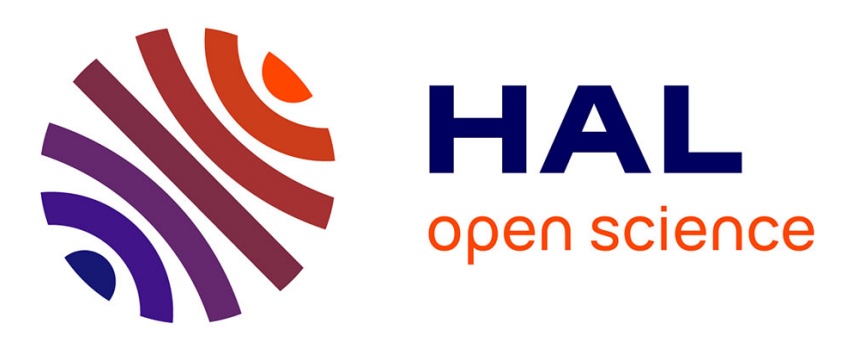

\title{
Isotopic composition of bare soil evaporated water vapor. Part II: Modeling of RUBIC IV experimental results
}

\author{
Isabelle Braud, Thierry Bariac, P. Biron, M. Vauclin
}

\section{To cite this version:}

Isabelle Braud, Thierry Bariac, P. Biron, M. Vauclin. Isotopic composition of bare soil evaporated water vapor. Part II: Modeling of RUBIC IV experimental results. Journal of Hydrology, 2009, 369, p. 17 - p. 29. hal-00547579

\section{HAL Id: hal-00547579 \\ https://hal.science/hal-00547579}

Submitted on 16 Dec 2010

HAL is a multi-disciplinary open access archive for the deposit and dissemination of scientific research documents, whether they are published or not. The documents may come from teaching and research institutions in France or abroad, or from public or private research centers.
L'archive ouverte pluridisciplinaire HAL, est destinée au dépôt et à la diffusion de documents scientifiques de niveau recherche, publiés ou non, émanant des établissements d'enseignement et de recherche français ou étrangers, des laboratoires publics ou privés. 


\section{Isotopic composition of bare soil evaporated water vapor.}

2 Part II: Modeling of RUBIC IV experimental results

3

4

5

6

7

8

9

10

11

12

13

14

15

16

17

18

19

20 21

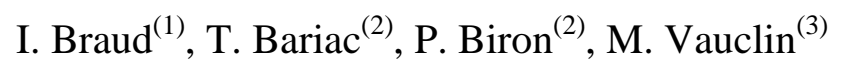

(1) Cemagref, UR HHLY, 3bis Quai Chauveau, 69336 Lyon Cedex 9, France

(2) BioEMCo (UMR 7618 CNRS, , ENS-Ulm, ENSCP, INRA, AgroParisTech, UPMC) INRA-INAPG, Bâtiment EGER 78850 Thiverval-Grignon, France

(3) LTHE (UMR 5564 CNRS, INPG, IRD, UJF), BP 53 X, 38041 Grenoble Cedex 9, France

9 Corresponding author: Isabelle Braud, Cemagref, UR HHLY, 3bis Quai Chauveau, 69336 20 Lyon Cedex 9, France, Tel: +33 4722087 78, $\underline{\text { isabelle.braud@cemagref.fr }}$ 


\section{Summary}

2 Stable water isotopes such as oxygen 18, are tracers of water movement within the soil -

3 vegetation -atmosphere system. They provide useful information for a better understanding of

4 evaporation and water vapor transport within soils. In part I of this paper, we presented a

5 novel control experimental set up under non steady conditions, dedicated to the measurement

6 of the evaporation flux and corresponding isotopic composition from six bare soil columns.

7 Data analysis raised several questions about the soil depth controlling the isotopic composition of the evaporated water vapor, suggesting different behavior before and after the appearance of back diffusion. Experimental data also suggested a time variable value of the kinetic fractionation factor. The present paper presents the modeling of the experimental results using the coupled heat, water and stable isotope transfer model SiSPAT_Isotope.

12 Model results were used for investigating the above questions more in details. For this purpose, model parameters were calibrated for each soil column in order to reproduce the data. Then model results were inverted to estimate the kinetic fractionation factor. The results show that the hypothesis that the kinetic fractionation factor varies in time is plausible but the uncertainty is too large to derive firm conclusions. The largest uncertainty is found when the soil relative humidity is lower than one but water vapor is still negligible. When back diffusion has occurred, model results are the most robust and confirm that the isotopic composition of the evaporated water vapor is controlled by the soil isotopic composition of the liquid water at the peak. In this case, the retrieved kinetic fractionation factor is close to $18.9 \%$, corresponding to laminar flow.

Keywords: Soil evaporation, Soil water, Water stable isotope, Kinetic fractionation factor, 


\section{Introduction}

3 Measurements of stable isotopic composition of soil water can provide useful information for

4 better quantifying water transfer within soils (mainly vapor diffusion coefficients and

5 hydraulic properties). We developed a physically based model, called SiSPAT_Isotope

6 (Braud et al., 2005a) for bare soil, representing the full interactions between the atmosphere,

7 the soil and stable isotope species. The model was evaluated against two sets of laboratory data (Braud et al., 2005b). The data were composed of soil columns which were let evaporating freely in the atmosphere. This first study evidenced lots of uncertainty in the modeling and interpretation of stable isotopic composition in terms of evaporation. The uncertainty was related to a lack of control of the experimental conditions, especially of the atmospheric relative humidity and to a lack of knowledge of the kinetic fractionation factor, which controls the isotopic flux close to the surface, for unsaturated soils. In Part I of this paper, we presented a novel control experimental set up, dedicated to the measurement of the evaporation flux and corresponding isotopic composition of evaporated water vapor from bare soil columns. The experiment allowed a more accurate determination of atmospheric conditions and evaporation flux than in previous studies. For the first time, the isotopic composition of soil evaporated water under quasi-saturated and unsaturated conditions was measured. In the present paper, the SiSPAT_Isotope model was used for interpreting the experimental results, focusing on the determination of the kinetic fractionation factor, as deduced from model results. Section 2 presents the model, the methodology retained to run it and calibrate its parameters, as well as the calculation of the kinetic fractionation factor from model results. Section 3 discusses the results and conclusions and perspectives are drawn in section 4. 


\section{Material and methods}

\subsection{The experimental set up}

The RUBIC IV reactor was developed in order to determine the isotopic composition of the water vapor released by an evaporating soil and to monitor its time evolution as long as the soil was drying. The leak tight experimental set up allowed us to send a dry air gas flow simultaneously over six evaporating soil columns and to trap continuously all the water vapor released by evaporation. The water samples were then analyzed using a mass spectrometer to determine their content in oxygen 18 . Therefore, the only water vapor source was that of the evaporating soil columns, without any contamination from external sources. Furthermore, a cryoscopic trapping ensured that all the water vapor coming from the soil evaporation was completely trapped. In the design of the RUBIC IV experimental set up (see Part I, Braud et al., submitted), the following constraints were taken into account:

- A continuous non destructive sampling for the water vapor phase and a destructive sampling for the liquid phase within the soil columns. It meant to dismantle the soil columns at successive dates, cut them into slices and analyze the isotopic composition of all samples, as well as their gravimetric water content

- A tight experimental set up with regards to external contaminations and loss of vapor or water from the system through a leak

- The continuous measurement and the regulation of climatic variables

- The continuous measurement of the most important variables describing the soil moisture status in the soil (water pressure and water content)

The experiment was launched on April 112005 (Day of the Year, DoY 101) and lasted 338 days. At successive dates during the drying process corresponding to respectively $31,53,91$, 163, 235 and 338 days of drying (DoD), one of the column was dismantled for analysis (Table 
1 1). All the details, including the description of the reactor and the measurements which were

2 carried out, are given in Part I of the paper (Braud et al., 2009)

3

\subsection{The SiSPAT_Isotope model}

The coupled heat, water and stable isotope transfer model (SISPAT_Isotope) was based on a Soil -Vegetation - Atmosphere Transfer (SVAT) model (SiSPAT, Braud et al., 1995; Braud, 2000; 2002). The bare soil version of the model was used in this study and the isotope transport module was extensively described in Braud et al. (2005a).

The isotope transport module is based on the mass conservation equation for the isotope species. Both the liquid and vapor concentrations as well as the liquid and vapor isotope fluxes are considered. The number of unknowns is reduced to the liquid isotope concentration only, by using the hypothesis of instantaneous equilibrium between the liquid and vapor phases. Details on the final set of equations, which can be written as a convection-diffusion type transport equation, as well as its numerical discretization are given in Braud et al. (2005a).

To close the system, upper and lower boundary conditions must be specified. For the bottom boundary condition, the user can chose between a constant value of the liquid water isotopic concentration or a bottom flux calculated as the product of the bottom liquid flux times the bottom liquid water isotopic concentration. For the upper boundary condition, the soil-atmosphere isotope flux was written as:

$$
E_{i}=\frac{\rho_{a}}{\alpha_{K} r_{a}} \frac{M_{i}}{M_{w}}\left(\alpha_{e}^{*} R_{i s}^{l} q_{s}-R_{i a}^{v} q_{a}\right)
$$

where $R_{i s}^{v}$ and $R_{i a}^{v}$ are the liquid isotopic ratio at the soil surface and the water vapor isotopic ratio in the atmosphere, respectively; $M_{i}(\mathrm{~kg})$ and $M_{w}(\mathrm{~kg})$ are the heavy and ordinary water molecular mass, respectively; $q_{s}$ and $q_{a}\left(\mathrm{~kg} \mathrm{~kg}^{-1}\right)$ are the specific humidity at the soil surface 
1 and in the atmosphere, respectively; $\rho_{a}\left(\mathrm{~kg} \mathrm{~m}^{-3}\right)$ is the air volumetric mass; $r_{a}\left(\mathrm{~s} \mathrm{~m}^{-1}\right)$ is the

2 aerodynamic resistance to heat and water vapor transfer; $\alpha_{e}^{*}(-)$ is the liquid-vapor isotopic

3 fractionation factor at equilibrium, and $\alpha_{K}(-)$ is the so-called kinetic isotopic fractionation

4 factor. Several formulae of this factor, taken from the literature were previously reviewed and

5 tested by Braud et al. (2005a) for synthetic data sets, showing a high sensitivity, which will be

6 investigated in this paper using experimental data and model results. The model is able to

7 perform calculation for oxygen 18 and deuterium. Only oxygen 18 was considered in this

8 paper.

9

10

11

12

13

14

15

\subsection{Model set up}

Each 1D soil column (12 cm in diameter, $35 \mathrm{~cm}$ of soil in height) was divided into 132 cells, with a logarithmic increase of the thickness from about $0.1 \mathrm{~mm}$ close to the surface and to the bottom and $5 \mathrm{~mm}$ in the center of the column. The soil was assumed homogeneous. The retention curve, relating the volumetric water content $\theta\left(\mathrm{m}^{3} \mathrm{~m}^{-3}\right)$ to the soil water pressure $h$ (m) was described by the Van Genuchten (1980) expression and the hydraulic conductivity $K$ $\left(\mathrm{m} \mathrm{s}^{-1}\right.$ ) by the Brooks and Corey (1964) equation, respectively:

$$
\frac{\theta}{\theta_{\text {sat }}}=\left(1+\left(\frac{h}{h_{\mathrm{VG}}}\right)^{n}\right)^{-m} \text { with } \quad m=1-\frac{2}{n}
$$

$$
K(\theta)=K_{\text {sat }}\left(\frac{\theta}{\theta_{\text {sat }}}\right)^{\eta}
$$

where $\theta_{\text {sat }}\left(\mathrm{m}^{3} \mathrm{~m}^{-3}\right)$ is the saturated water content, $h_{V G}(\mathrm{~m})$ is a scaling parameter for soil water pressure, $K_{\text {sat }}\left(\mathrm{m} \mathrm{s}^{-1}\right)$ is the saturated hydraulic conductivity and $m, n$, and $\eta$ are shape parameters related mainly to soil texture (Haverkamp et al., 2005). In addition, soil water vapor diffusivity depends on the soil tortuosity $\tau(-)$ which is another parameter of the model. The residual water content was set at zero, which is consistent with the measurements 
1 (minimum measured value was $0.01 \mathrm{~m}^{3} \mathrm{~m}^{-3}$ ). The saturated water content was also known

2 from the dry bulk density and initial water volume used to saturate each column from their

3 base (see Table 2 in Braud et al., 2009 for details). For the retention curve of Eq. (2), the

4 simultaneous measures of soil water pressure $h$ and volumetric water content $\theta$ at $-2.5 \mathrm{~cm}$

5 depth were used to fit the value of the $h_{V G}$ and $n$ parameters (see Fig. 5 in Braud et al., 2009).

6 The other parameters were considered for optimization (see below).

7 To run the model, initial and boundary conditions must also be specified. They are given in

8 Table 2. Let's concentrate on the upper boundary condition. In the original model (Braud et

9 al, 2005a), surface evaporation and isotope fluxes were calculated from the climatic

10 conditions at a reference level and the surface conditions by using a resistance type approach.

11 In the context of the RUBIC IV experiment, these fluxes were measured. The first idea was to 12 force the model using these measured values of the fluxes. However, we found that, if the soil 13 hydraulic properties were not correctly prescribed, numerical instability could be generated in

14 the model, especially when it was forced by evaporation fluxes the soil was not able to

15 provide. This solution could therefore not be used in the optimization process, to avoid failure

16 of the algorithms. For the consistency of the model in the optimization procedure, the model

17 was let calculate the evaporation flux using the following formula:

$$
E=\rho_{a} \frac{q_{s}-q_{a}}{r_{a v}}
$$

$\rho_{a}$ and $q_{a}$ being deduced from the measured air temperature and humidity at the outlet of the column, which can be assumed to be representative of the conditions prevailing above the soil surface (Braud et al., 2009); $q_{s}$ was calculated by the model. The unknown was therefore the aerodynamic resistance $r_{a v}$. Unfortunately, the wind speed above the column was not measured continuously. Assumptions were therefore required for the estimation of the resistance. They are detailed hereinafter in the description of the optimization process. Once 
1 the parameters were optimized, the model was also run by forcing it with the measured values

2 of the evaporation flux, to verify the consistency of the results.

3 When running the isotope transport module, the measured isotopic composition $R_{\text {iout }}^{v}$ of the

4 outlet water vapor was used and the surface isotope flux was forced as:

$$
E_{i}=\frac{M_{i}}{M_{w}} R_{\text {iout }}^{v} E
$$

Another method could have been used to provide the isotope transport module surface boundary condition, at least before the appearance of back diffusion. Equation (15) of Part I (Braud et al., 2008) could have been used to estimate the soil surface liquid isotopic composition from the measured isotopic composition of the evaporated water vapor. However this solution was not retained as (i) it required the choice of a value for the kinetic fractionation factor, we wanted to estimate independently and (ii) it was not applicable for the whole experiment.

\subsection{Optimization of parameters of the water and heat transfer modules}

The first application of the SiSPAT_Isotope model presented in Braud et al. (2005b) showed that a proper simulation of water transport was a prerequisite to a correct simulation of stable isotope concentrations. As the major objective of the present study was to understand the processes involved in evaporation, we chose first to optimize the missing soil and aerodynamic parameters in order to reproduce the observations as well as possible. In a second step, this allowed us to use model results for testing various hypotheses about active processes and for interpreting and deriving the kinetic fractionation factor. For the optimization, the HAUS59 software, based on the iterative Gauss-Marquardt method and the maximum likelihood criterion (Bard, 1974) was used. The optimization function $F\left(P_{\text {aram }}\right)$ was defined as follows: 


$$
F\left(P_{\text {aram }}\right)=\sum_{i=1}^{n} \sum_{k=1}^{5}\left(\frac{y_{i}^{\bmod k}\left(P_{\text {aram }}\right)-y_{i}^{\text {obsk }}}{\bar{y}_{\text {obs }}^{k}}\right)^{2}
$$

2 where $y_{i}^{\operatorname{modk}}\left(P_{\text {aram }}\right)$ are the model values as function of the parameter set $P_{\text {aram }}$ and $y_{i}^{\text {obsk }}$ are

3 the corresponding observations. The $k$ index refers to the various series which were used. For

4 column 1, the series of volumetric water content $\theta$ at depths $-2.5,-8,-16$ and $-24 \mathrm{~cm}$ and the

5 evaporation flux $E\left(\mathrm{~kg} \mathrm{~m}^{-2} \mathrm{~s}^{-1}\right)$ were used. For the other five soil columns, only the

6 evaporation flux was available. As the observed variables were of different nature, the

7 differences between modeled and observed values were normalized using $\bar{y}_{\text {obs }}^{k}$, the average of

8 each observation series. Indices $i=1$, n refer to the various time steps where observations were

9 available. For the efficiency of the optimization, we used daily average values in Eq. (6),

10 although the original observations were hourly values.

11 The optimization process was conducted in several steps, using only the coupled heat and

12 water transport modules (without isotopes). Several strategies were tested on column 1 (which

13 was provided with the largest number of observations and the longest time series, see Table 1)

14 before the final strategy, presented below was retained.

15 In a first step, we tried to optimize three soil parameters, namely $K_{s a t}, \eta$ and $\tau$ as well as a

16 constant value of the aerodynamic resistance $r_{a v}$ as used by Schneider et al. (2006). We also

17 tested an optimization including $h_{V G}$ and $n$, but the algorithm results showed that these

18 parameters were correlated with $K_{\text {sat }}$ and $\eta$, without significant gain in the optimization

19 function, but with much less robustness in the optimized parameters. Therefore the values of

$20 h_{V G}$ and $n$, fitted on the measurements, were used in the remainder of the study. Figure 1.

21 shows the results of this first optimization for column 1 . When using a constant aerodynamic

22 resistance, Fig. 1 (top) shows that the calculated time series of the evaporation flux was not

23 consistent with the measured one. Furthermore, Figure 1 (bottom) shows that the aerodynamic

24 resistance, calculated by inverted Eq. (4) using the measured $E$ and $q_{s}$ calculated by the model 
1 was not constant, as assumed in the optimization. Contrarily to the experimental conditions of

2 Schneider et al. (2006), it was thus not possible to assume that the aerodynamic resistance

3 was constant during the whole experiment. Figure 1 (bottom) suggests that the aerodynamic

4 resistance can be approximated by straight lines segments. Except for the first one, the dates

5 at which the slope of the lines was changing were corresponding with the dates where the

6 constraints imposed on the air relative humidity $\left(h_{a}\right)$ above the soil columns were modified as

7 well (Table 3). As explained in Part I of the paper (Braud et al., 2009), it was necessary to

8 modify the constraint on the relative air humidity above the soil column to fulfill experimental

9 constraints on the maximum value of the dry air flux flowing above the columns. The change

in relative air humidity had of course an impact on the evaporation flux and soil isotope

composition profiles. However, all these changes were monitored and taken into account

12 directly into the modeling though the use of the measured evaporation flux and isotopic composition of the evaporated water vapor as surface boundary conditions.

A second set of optimization was therefore realized using the function given by Eq. (7) for the

following three resistances $\mathrm{r}_{\mathrm{av} 0}, \mathrm{r}_{\mathrm{av} 1}, \mathrm{r}_{\mathrm{av} 2}$ were considered for optimization.

19

$$
\left\{\begin{array}{l}
r_{a v}(t)=r_{a v 0}+\frac{\left(r_{a v 1}-r_{a v 0}\right)}{t_{1}} t \quad 0 \leq t \leq t_{1} \\
r_{a v}(t)=r_{a v 1}+\frac{\left(r_{a v 2}-r_{a v 1}\right)}{\left(t_{2}-t_{1}\right)}\left(t-t_{1}\right) \quad t_{1} \leq t \leq t_{2} \\
r_{a v}(t)=r_{a v 2} \quad t_{2} \leq t \leq t_{3}
\end{array}\right.
$$

Several trials and errors were conducted to determine the sensitive parameters and get a robust optimization. They showed that only the Brooks and Corey soil parameter, $K_{\text {sat }}$ (Eq. (3)) was independent and the other one, $\eta$, was fixed at $\eta=6.67$. In the same way, the soil 
1 tortuosity was fixed at $\tau=0.4$. Finally, only the three aerodynamic resistances and the

2 saturated hydraulic conductivity were optimized. The optimization algorithm was run several

3 times with different initial values of the corresponding parameters in order to control that the

4 model had reached the absolute optimum. For the columns with the shortest time series

5 (columns 5 and 6), the evaporation flux was found to be quite insensitive to the saturated

6 hydraulic conductivity (evaporation was more atmosphere controlled than soil controlled

7 during these periods). Therefore, the $K_{\text {sat }}$ value of column 1 was used and only the first two

8 aerodynamic resistances ( $r_{a v 0}$ and $\left.r_{a v 1}\right)$ were optimized (see Table 4). After the optimization,

9 the model was run using the measured evaporation flux as the upper boundary condition with

10 the optimized soil parameters in order to verify the consistency of the results.

11

\subsection{Calibration of the isotope transport module}

Results of Braud et al. (2005a, b) have shown that the value of the soil tortuosity $\tau$ was tightly related to the depth of the peak within the isotope concentration profiles. The value $\tau=0.4$ which was retained in section 2.4 was fulfilling this requirement.

A second parameter to be specified in the isotope transport module is the dispersivity length $\Lambda$ (m) which appears in the total liquid diffusivity $D_{i}^{l^{*}}\left(\mathrm{~m}^{2} \mathrm{~s}^{-1}\right)$ for isotope $i$. It is given by Eq. (8) where $D_{i}^{l o}\left(\mathrm{~m}^{2} \mathrm{~s}^{-1}\right)$ is the molecular diffusivity of isotope $i$ in liquid water, $\theta_{l}\left(\mathrm{~m}^{3} \mathrm{~m}^{-3}\right)$ is the volumetric water content and $q_{l}\left(\mathrm{~m} \mathrm{~s}^{-1}\right)$ is the liquid water flux.

$$
D_{i}^{l^{*}}=D_{i}^{l o} \tau \theta_{l}+\Lambda\left|q_{l}\right| / \theta_{l}
$$

The sensitivity of model results to this parameter was first investigated. Then, the value was calibrated by hand for each column, by matching observed and simulated maximum or surface isotopic composition within the soil profiles (see section 3.2). 
2 In Part I of the paper, Braud et al. (2009) showed that, given the experimental conditions and

3 the expression of the surface isotopic flux (Eq. (1)), the kinetic fractionation factor can be

4 retrieved as:

$5 \quad \alpha_{K}=\frac{\left(\alpha_{e}^{*} R_{\text {is }}^{l} q_{s}-R_{\text {iout }}^{v} q_{a}\right)}{\left(q_{s}-q_{a}\right) R_{\text {iout }}^{v}}$

To apply Eq. (9), we used the measured data for the specific humidity of the air above the soil

7 column $q_{a}$, and its isotopic composition $R_{\text {iout }}^{v}$, as well as the values calculated by the model for

8 the soil surface specific humidity $q_{s}$ and the isotopic composition of surface liquid water

9 content $R_{i s}^{l}$.

As the depth controlling the kinetic fractionation within the soil profile remains unknown for unsaturated soils, additional calculations were performed using the specific humidity $q_{s_{-} \text {front }}$ and the isotopic composition of the soil liquid content $R_{i s_{-} \text {front }}^{l}$ at the evaporation front. The latter can easily be identified within the soil profile as it corresponds to the depth at which liquid and vapor fluxes are equal (Barnes and Allison, 1983; Braud et al., 2005a). This depth also corresponds to the depth of the peak within the soil isotopic composition profile. The appearance of this peak is referred to as the appearance of back diffusion in the remainder of the paper. The concept of evaporation front appears in modeling approaches of evaporation proposed by Bastiaanssen et al. (1989) and revisited by Gowing et al. (2006). The soil humidity controlling evaporation is taken at the evaporation front with a soil resistance $r_{\text {soil }}$ added to the aerodynamic one to take into account the resistance to water vapor transfer

21 within the dry soil layer. Therefore, Eq. (4) can be rewritten as:

$$
E=\rho_{a} \frac{q_{s_{-} \text {front }}-q_{a}}{r_{a v}+r_{\text {soil }}}
$$


1 where the subscript _front refers to the value at the depth of the evaporation front. The inversion

2 of this equation allows the determination of $r_{a v}+r_{\text {soil }}$.

3 For the isotopic surface flux, the analogy is more complicated. Between the evaporation front

4 and the surface, the isotope flux occurs mostly in the vapor phase. Braud et al. (2005a)

5 defined the vapor isotopic flux within the soil, $q_{i}^{v}\left(\mathrm{~kg} \mathrm{~m}^{-2} \mathrm{~s}^{-1}\right)$, as:

$6 \quad q_{i}^{v}=C_{i}^{v} q_{v}-D_{i}^{v^{*}} \frac{\partial C_{i}^{v}}{\partial z}$

7 where $q_{v}\left(\mathrm{~m} \mathrm{~s}^{-1}\right)$ is the vapor water flux, $D_{i}^{v^{*}}$ is the total vapor diffusivity $\left(\mathrm{m}^{2} \mathrm{~s}^{-1}\right)$ for isotope $i$.

$8 C_{i}^{v}\left(\mathrm{~kg} \mathrm{~m}^{-3}\right)$ is the vapor isotopic concentration, which is related to the liquid concentration

$9 \quad C_{i}^{l}$ by:

$$
C_{i}^{v}=\frac{M_{i}}{M_{w}} R_{i}^{v} \rho_{v}=\frac{M_{i}}{M_{w}} \alpha_{e}^{*} R_{i}^{l} \rho_{v}=\alpha_{e}^{*} \frac{\rho_{v}}{\rho_{w}} C_{i}^{l}=\beta_{i}^{*} C_{i}^{l}
$$

$11 \quad$ with $\beta_{i}^{*}=\alpha_{e}^{*} \frac{\rho_{v}}{\rho_{w}}$

12 and $\alpha_{e}^{*}(-)$ is the liquid-vapor isotopic fractionation factor at equilibrium given by Majoube

13 (1971) as a function of temperature $T(\mathrm{~K}), \rho\left(\mathrm{kg} \mathrm{m}^{-3}\right)$ is the volumetric mass of water either in 14 the liquid, $\rho_{w}$, or in the water vapor phase, $\rho_{v}$.

15 If the diffusive term of the isotopic vapor flux is negligible as compared to the convective 16 term in Eq. (11), and if the vapor flux is uniform between the evaporation front and the 17 surface, thus, the isotopic composition of the evaporated water vapor would not be modified very much between the evaporation front and the surface. Eq. (9) can thus be applied with the

19 values at the evaporation front and the estimation of the kinetic fractionation factor $\alpha_{i K}$

20 becomes:

$$
\alpha_{K}=\frac{\left(\alpha_{e}^{*} R_{\text {is_front }}^{l} q_{s_{-} \text {front }}-R_{\text {iout }}^{v} q_{a}\right)}{\left(q_{s_{-} \text {front }}-q_{a}\right) R_{\text {iout }}^{v}}
$$


1 Eqs. (9) and (14) should lead to the same results before the appearance of back diffusion.

2 Hypotheses leading to Eq. (14) are consistent with data analysis performed by Braud et al.

3 (2009) suggesting that the isotopic composition of the evaporated water could be controlled

4 by the peak liquid concentration within the soil profile. The validity of this hypothesis will be

5 evaluated in the next section.

6

7

8

9

\section{Results}

\subsection{Optimization of parameters and simulation of water transfer}

Values of the optimized parameters are given in Table 4, together with their estimated confidence interval, provided by the optimization algorithm. The figures show that most of the parameters are estimated with a narrow uncertainty, except the third resistance $r_{a v 2}$. The uncertainty on the third resistance $r_{a v 2}$ is larger for columns 2 and 4 because only the evaporation flux time series was used in the optimization, whereas both evaporation flux and soil moisture time series were used for column 1 . Table 5 shows the correlation between the estimated values (Eq. (4) with the optimized resistances) and observed hourly values of the evaporation flux for all the columns. Figure 2 provides an example of the time series for columns 1 and 5. Results are much more satisfactory for the longest drying periods. The measured evaporation flux exhibits high frequency fluctuations, certainly related to time variations in the air temperature, which contrarily to the air relative humidity was not regulated. These fluctuations made the modeling and data interpretation more difficult. Furthermore, simulations were performed assuming vertically homogeneous soil profiles, whereas the real ones certainly exhibit some vertical heterogeneity due to heterogeneities in the soil filling and saturation. 
1 Subsequent results analysis shows that it was relevant to optimize a parameter set for each

2 soil column in order to get the best estimates of the evaporation flux. Indeed, model results

3 interpretation in terms of isotope transfer appear very sensitive to the values of this flux.

$5 \quad$ Figure 3 and Table 5 provide the comparison between measured and simulated soil water

6 content profiles both using the evaporation flux calculated by Eq. (4) and the measurement

7 flux as surface boundary condition. The agreement is satisfactory, especially for the last

8 profiles. When using the measured evaporation flux, results were very similar to those

9 obtained by Eq. (4), except for column 3 (91 DoD, DoY 192) where the drying is much less when using the measured evaporation flux (77.2 mm versus $85.4 \mathrm{~mm})$. The comparison of cumulated evaporation derived from three methods exposed in Braud et al. (2009) showed cumulative values varying between 77.2 and $80.4 \mathrm{~mm}$. However, the simulated profile is closer to the observed one using Eq. (4). For column 4 (235 DoD, DoY 336), the model diverged when using the measured flux, due to an evaporation flux larger than the value the soil was able to provide. In the following, results of column 4 were discarded as they were inconsistent, putting some doubts on the quality of the measurements. The comparison of the simulations using the evaporation flux calculated by Eq. (4) and the measurement flux as surface boundary condition, shows that the hypotheses on the aerodynamic resistances and the parameter optimization were reasonable.

\subsection{Simulation of isotope concentrations profiles}

Figure 4 shows the comparison of simulated and observed oxygen 18 soil water isotopic composition profiles of columns 5 (53 DoD, DoY 154), 2 (163 DoD, DoY 264) and 1 (338

DoD, DoY 439) for various values of the dispersivity length $\Lambda$ ranging between 0 and $0.05 \mathrm{~m}$. These columns are representative of the results obtained before (column 5) and after (columns 
12 and 1) the establishment of a back diffusion profile. It appears that model results are very

2 sensitive to the value of the dispersivity length. An increase in $\Lambda$ leads to a decrease of the

3 peak value and conversely. Results also show that a zero value of $\Lambda$ leads to a large

4 overestimation of the peak, except for column 4 (see Fig. 5) where it was still underestimated

5 with $\Lambda=0$. Before back diffusion occurs, model results are very sensitive to the dispersivity

6 length with a range of about $15 \%$ for the surface values (column 5). The sensitivity of the

7 peak value is smaller after the establishment of back diffusion and decreases as long as the

8 drying period length increases: the range is about $10 \%$ for column 2 and $5 \%$ for column

9 1. The range of the simulated surface values is larger than for the peak (about $10 \%$ ) for

10 these two columns.

11 After the establishment of back diffusion, it was not clear if the optimization of $\Lambda$ should

12 favor a better representation of the surface or of the peak values (as it was not possible to get a

13 value leading to a correct simulation of both, except for column 2). The retained optimal

14 value for the dispersivity presented in Table 4 for column 1 was chosen so that the surface

15 value was correctly simulated, although it was leading to an overestimation of the peak value

16 (Fig. 4, right).

17 Table 4 provides the optimized values of $\Lambda$ for the six columns. Because dispersivity is well-

18 known to be sensitive to heterogeneities at small scales, this may explain the more or less

19 erratic values obtained for the various columns and the absence of a logical evolution amongst

20 them. For column 4, even a zero value provides an underestimation of the peak and very low

21 values close to the surface. As mentioned before, the results of this column are thought to be

22 non reliable. For column 1, none of the values of the dispersity was leading to a correct

23 simulation of both the surface and peak value, whereas it was possible for column 2, but with

24 a very large value of the dispersity. It must be mentioned that the sampling of column 1 was

25 very difficult because the soil was very dry and very friable. Furthermore, as the water content 
1 close to the surface was very low, the measure of the oxygen 18 isotopic composition is

2 probably less reliable than for column 2, as can be seen from the shape of the profile which is

3 much more irregular.

4 Figure 5 shows the simulated oxygen 18 composition soil profiles obtained using the

5 “optimal” value of the dispersivity provided in Table 4 for each soil column and an

6 evaporation flux either calculated using Eq. (4) or measured. They are compared with the

7 observations. Table 5 provides the statistics between the simulated and observed profiles in

8 both cases. The agreement is very good for columns 6, 5, 2 and 1 ( $R^{2}$ larger than 0.95$)$ and

9 results are similar for the two choices of the boundary conditions, with a slight decrease in

10 performance when the measured evaporation flux was used. For column 3, model

11 performance is poorer $\left(R^{2}=0.90\right.$ with a 0.73 slope) with a large decreases in statistical criteria

12 when the measured evaporation was used, corresponding to an underestimation of the surface

13 values. It must be related to the slower drying mentioned in the previous section. As for water

14 content, we noticed a divergence of the model for column 4 forced by the measured

15 evaporation flux.

16

\subsection{Simulation of water and isotope fluxes}

In order to get insight into the model results, we investigated possible processes which were involved and verified if hypotheses stated in section 2.6 hold. Figure 6 shows the liquid, vapor and total fluxes of oxygen 18 for columns 5, 2 and 1. It can be seen that below the peak of oxygen 18 isotopic content (located at the depth where liquid and vapor fluxes are equal, Barnes and Allison, 1983; Braud et al., 2005a,b), liquid isotope (and water, not shown) flux is dominant whereas above the peak, vapor flux is dominant. Figure 6 also shows that the columns are not in a stationary state, as the total isotope flux increases from zero at the bottom to a maximum value at the surface (column 5). When water vapor flux is dominant 
1 (dashed lines in Fig. 6), the isotope flux is almost constant between the soil surface and the

2 depth of the peak of oxygen 18 isotopic composition (columns 2 and 1). Thus, one of the

3 assumptions made in section 2.6, is shown to hold. This non stationary characteristic of the

4 experimental set up is important to keep in mind, as the traditional results in isotopic

5 geochemistry, based on isotopic stationary states hypotheses, do not hold. The results

6 obtained after the establishment of back diffusion can corroborate the findings of

7 Saravanapavan and Salvucci (2000). They showed that, when the evaporation flux was

8 limited by the capacity of capillary rises to provide the water, the depth of the evaporation

9 front was adapting to allow the soil to transfer the water flux from deeper layers towards the

10 surface. Our experimental and modeling results confirm this finding as the vapor flux is

11 almost constant between the evaporation front and the soil surface.

12 Figure 7 (left) provides the ratio between the diffusive and total liquid oxygen 18 flux. This

figure shows that, when the liquid flux is dominant (i.e. below the peak), the diffusive component of the flux is only a few percents of the total flux, which is dominated in this case by convective transport. This fraction increases from the bottom towards the top of the profile, up to the depth of the maximum oxygen 18 content. The diffusive flux is directed downwards whereas convective flux is directed upwards (not shown). Due to this opposite sign, the enrichment of the isotopic profile close to the surface is limited. The relative contribution of the diffusive part of the flux increases as long as the soil dries (Fig. 7 shows an increasing contribution from column 5 to 2 and 1).

The analysis of the vapor isotope flux (Fig. 7, right) only makes sense after the establishment of back diffusion, i.e. for columns 2 and 1 (and above the peak). The contribution of the diffusive part to the total vapor isotope flux is still restricted to a few percents. This confirms the hypothesis made in section 2.6, that convective transport of water vapor should be dominant between the evaporation front and the surface. Thus the isotopic composition of the 
1 evaporated water should be related to the isotopic composition of the soil liquid water at the

2 peak. From this analysis of the model results, it can be concluded that Eq. (14) should be

3 suited to derive an estimation of the kinetic fractionation factor, as will be shown in the

4 following section.

5

\subsection{Kinetic fractionation factor}

Figure 8 shows the calculated kinetic fractionation factor before the establishment of back diffusion (columns 6, 5, 3) (top) and the corresponding soil surface relative humidity (bottom). The retrieved values are very different from one column to the other. Column 5 was exhibiting the most consistent results when the simulation was performed either using Eq. (4) or the measured values and can be considered as the most reliable column. For all the columns, the calculated range for the kinetic fractionation factor is larger than the range reported in the literature and the estimation can sometimes become negative (column 3). Apart from the differences in absolute values, Figure 8 shows that the three columns exhibit similar behaviors: larger values are calculated at the very beginning of the experiment, but results are certainly less reliable due to time required by the system to reach its equilibrium. Then, the kinetic fractionation factor seems to stabilize (up to about DoY 150) around a value which remains very uncertain, and then begins to decrease to reach smaller values than those given in the literature. Braud et al. (2009) show that an isotopic stationary state was noticeable before DoY 150 (Fig. 9 in Part I) and it corresponds to a relative humidity very close to 1 . The calculated kinetic fractionation factor decreases when the soil surface relative humidity begins to decrease, although the latter only reaches a value of 0.9975 . Figure 8 seem to confirm one of the hypotheses raised in Braud et al. (2009) from the data analysis that the kinetic fractionation factor could vary in time. Stable values are obtained when the relative 
1 humidity is close to one, and then the kinetic fractionation factor seems to decrease, even

2 below values reported in the literature.

3 Figure 9 provides the calculated kinetic fractionation factor for columns 2 and 1 for the

4 optimum dispersivity length (0.046m for column 2 and $0.055 \mathrm{~m}$ for column 1 - see Table 4$)$

5 and a value of $0.01 \mathrm{~m}$. For both cases, Figure 9 gives estimates derived from Eq. (9), i.e. using

6 the calculated soil surface value (full line) and Eq. (14), i.e. using the calculated values at the

7 evaporation front (dashed line). For column 2, Figure 9 (top) shows that the calculated kinetic

8 fractionation factors are very low and part of the time negative when using the optimum

9 dispersivity of Table 4. More realistic values were obtained with a dispersivity of $0.01 \mathrm{~m}$, which was leading to an overestimation of the isotopic peak value (see Fig. 4). For column 1 and the optimized dispersivity $(0.055 \mathrm{~m})$, the calculated kinetic fractionation factor falls in the range of literature values. For both columns, the shape of the curve is similar to that of columns 6, 5, 3 (Fig. 8, top) at the beginning of the drying process. When Eqs. (9) and (14) provide different values, it means that back diffusion process has begun to occur. It is remarkable that, for column 1 , the calculated kinetic fractionation factor is very stable after back diffusion, whatever the value of the dispersivity is, and converges towards a kinetic fractionation factor of 18.9 (per mil), when using Eq. (14) (Fig. 9 bottom, dashed lines). This result confirms the hypothesis of an isotopic composition of the water vapor controlled by the value of the isotopic composition of the soil liquid water at the evaporation front after back diffusion has taken place. On the other hand, the use of Eq. (9) and of the surface value to retrieve the kinetic fractionation factor leads to lower values than in the literature (full lines Fig. 9 bottom) and the result depends on the dispersivity length. This result shows that the use of Eq. (26) given in Braud et al. (2005a):

$$
E_{i}=\frac{\rho_{a}}{\alpha_{K} r_{a}} \frac{M_{i}}{M_{w}}\left(R_{i s}^{v} q_{s}-R_{i a}^{v} q_{a}\right)
$$


1 to derive the surface isotopic flux, might either not be correct when back diffusion has

2 occurred, or the formulation of the isotope transport in the vapor phase might be deficient.

3

\subsection{Discussion}

The results presented in the paper show that experimental data analysis and modeling are complementary. Data analysis allow to raise hypothesis about processes. These hypotheses can be tested using the model which also provides a way to "interpolate" between measurements. Model results also give access to variables which cannot be measured such as water and isotope fluxes and which are important to understand the processes. Indeed, in Braud et al. (2009), data analysis allowed raising some hypotheses about the processes involved in soil evaporation and the isotopic composition of the evaporated water vapor. These hypotheses were that (i) the isotopic signature of soil evaporation (under non stationary conditions) might be controlled by the value of the isotopic content at the depth of the evaporation front and (ii) the kinetic fractionation factor was not constant during the whole drying process. The modeling results presented in this paper allowed partly confirming these hypotheses. The results rely on a careful calibration of the water transport module, using measured evaporation flux and hypotheses about aerodynamic resistances. Then, sensitivity tests were performed to assess the impact of the dispersivity length on the simulated oxygen 18 profiles. Although this parameter was optimized, it remains largely uncertain due to uncertainties on the measured soil oxygen 18 profiles, evaporation fluxes and isotopic composition of the evaporated water vapor. Therefore, the retrieved kinetic fractionation factors are not reliable in absolute values, except when the back diffusion is well established and the evaporation flux is located several cm below the soil surface (column 1). It is remarkable that all the columns show the same characteristic time evolution of this factor. Larger values are retrieved at the beginning of the drying process, when the soil surface 
1 relative humidity is close to one. When the soil surface dries and back diffusion is not

2 established, the kinetic fractionation factor decreases, even towards values lower than those

3 reported in the literature. It is certainly the period, where our conclusions are the less certain.

4 Then, when back diffusion has established, the retrieved kinetic fractionation factor is quite

$5 \quad$ stable and the calculated value is close to 1.0189 for oxygen 18.

6 As far as modeling is concerned, the surface isotopic flux was calculated using the measured

7 isotopic signature of the water vapor (see Eq. (5)). If the latter was not known, Eq. (15)

8 should be used instead. But the difference between Eqs. (9) and (14) in terms of retrieved

9 kinetic fractionation factor, shows that this formula should be used with lower values than those of the literature and our results do not tell us how it could vary and how it could be

11 formulated. Eq. (15) with values taken at the evaporation front could be used, but it is not 12 clear if it would be consistent with the model formulation of vapor isotopic transfer. As far as field studies are concerned, non stationary isotopic situations are likely to be encountered. The experiment results reported in Part I (Braud et al., 2009) and in this paper show that the traditional method which derives the isotopic composition of evaporation from the Craig and Gordon (1965) formula and the sampling of an arbitrary thickness (i.e. Yakir and Sternberg, 2000) for the soil surface isotopic composition is not correct when back diffusion occurs. In the absence of back diffusion, we have shown that the isotopic composition of the evaporated water vapor is controlled by the value within a very thin soil surface layer, which is almost impossible to sample. The traditional method is therefore likely to underestimate the isotopic composition of evaporated water vapor. Furthermore, our results show that the picture is much more complex and further efforts in modeling and results analysis are required to improve the modeling approach and/or better assess the dependency 


\section{Conclusions and perspectives}

3 This paper and Part I (companion paper by Braud et al., 2009) investigated the isotopic composition of bare soil evaporated water vapor. A novel controlled experiment was set up, which allowed measuring both the evaporation flux and its isotopic composition for six columns. Model results analysis showed that, although the air relative humidity was regulated, the variations in the air temperature were leading to fluctuations in the measured fluxes, which were complicating data and model analysis. It would be advisable to perform again the experiments by regulating the air temperature, to avoid diurnal fluctuations in the evaporation flux. As much as possible, the set up should be improved to get a more constant value of the air relative humidity. The experimental design allowed measuring the isotopic composition of evaporated water vapor with a good accuracy. However, the measurements of the soil isotopic composition of the liquid water profiles remain quite uncertain, due to difficulties in sampling a very fine thickness of soil. A better accuracy would have possibly been obtained by freezing the soil columns.

From the RUBIC IV experiment and the subsequent modeling exercise, it can be concluded that for evaporating unsaturated soils, the isotopic enrichment of the liquid water within the soil is dependent on the kinetic fractionation factor. The value of this factor is suspected not to be constant in time but the accuracy of the experiment was not sufficient to draw firm conclusions about this hypothesis. Smaller values below those reported in the literature seem to be possible. The experiment allowed drawing reliable conclusions for very dry soils with a well established back diffusion. In this case, the composition of evaporated water vapor is controlled by the isotopic concentration at the depth of the peak and the kinetic fractionation factor is close to 1.0189, i.e. the Merlivat (1978) value for laminar flux. When back diffusion has not occurred and the soil has dried enough to have a relative humidity significantly 
1 smaller than 1 , the experiment set-up did not allow complete understanding of all the

2 processes involved in the isotopic composition of evaporated water vapor. Nevertheless, the

3 experimental design appears to be promising but should be improved as stated above to allow

4 deriving less uncertain conclusions in this case.

5 The study we have performed was restricted to controlled conditions, because it was the only

6 way to isolate the isotopic composition of soil water from external contaminations. Under

7 field conditions, the Keeling plot (Keeling, 1961) showing the isotopic composition of the

8 water vapor as a function of the water vapor isotopic composition (values are measured at

9 various altitudes from the soil surface up to about $10-12 \mathrm{~m}$ height) is able to give elements

10 showing the possible impact of soil evaporation on the isotopic composition of the air water

11 vapor. If the soil surface is bare, the ordinate at the origin provides the isotopic composition

12 of the evaporated water. This value can be compared with the use of the Craig and Gordon

13 model which must be applied with caution. Our results show that the application of this

14 formula using the isotopic composition of the soil water averaged over several $\mathrm{cm}$ thickness is

15 likely to be underestimated, leading to biased estimation of the partition between bare soil

16 evaporation and regional contribution.

17 In the presence of vegetation, the picture is more complex. If there are only two sources of water vapor (regional and transpiration) the ordinate at the origin should be the isotopic composition of transpired water (itself being equal to that of the tiller of the plants under stationary isotopic conditions). It this hypothesis does not hold, thus a contribution from bare

21 soil evaporation must be invoked. In general the isotopic composition of the evaporation 22 water vapor is obtained from the Craig and Gordon model. As before, our results show that 23 the use of this formula using the isotopic composition of the soil water averaged over several $24 \mathrm{~cm}$ thickness is likely to be underestimated, leading to biased estimation of the partition 25 between bare soil evaporation and transpiration. 


\section{Acknowledgements}

The "Programme National de Recherche en Hydrologie" of the French "ECosphère

COntinentale" program is acknowledged for providing the financial support of the

experiment. A. Chanzy is thanked for providing the HAUS59 optimization code.

\section{References}

Bard, A., 1974. Non-linear parameter estimation. Academic Press, New-York.

Barnes, C.J. and Allison, G.B., 1983. The distribution of deuterium and 180 in dry soils: 1.

Theory. J. Hydrol. 60, 141-156.

Bastiaanssen, W. G. M., Kabat, P., Menenti, M., 1989. A new simulation model of bare soil evaporation in arid regions (EVADES). Instituut voor Cultuurtechniek en Waterhuishouding (ICW), Wageningen, Netherlands.

Braud, I., 2000. SiSPAT users’s manual, version 3.0., 106 pp.

Braud, I., 2002. SiSPAT User’s Manual Update, version 4.0, 13 pp.

Braud, I., Biron, Ph, Bariac, T., Richard, P., Canale, L., Gaudet, J.P., Vauclin, M., 2009.

Isotopic composition of bare soil evaporated water vapour. Part I: Experimental setting and results. Journal of Hydrology, submitted.

Braud, I., Bariac, T., Gaudet, J.P., Vauclin, M., 2005a. SiSPAT_Isotope , a coupled heat, water and stable isotope (HDO and $\mathrm{H}_{2}{ }^{18} \mathrm{O}$ ) transport model for bare soil. Model description and first verification. J. Hydrol. 309, 277-300.

23 Braud, I., Bariac, T., Vauclin, M., Boujamlaoui, Z., Gaudet, J.P., Biron, Ph., Richard, P., 
1 model for bare soil. Evaluation and sensitivity tests using two laboratory data sets. J. Hydrol. 2 309, 301-320.

3 Braud, I., Dantas-Antonino, A.C.,Vauclin, M., Thony, J.L., Ruelle, P., 1995. A Simple Soil

4 Plant Atmosphere Transfer model (SiSPAT). Development and field verification. J. Hydrol. 5 166, 213-250.

6 Brooks, R.H., Corey, A.T., 1964. Hydraulic properties of porous media. Hydrology paper, 3, 7 Colorado State University, Fort Collins, 27 pp.

8 Craig, H. Gordon, L.I., 1965. Deuterium and oxygen 18 variations in the ocean and the 9 marine atmosphere, Proceedings of the Conference on the stable isotopes in oceanographic studies and paleotemperatures, Lab. Geol. Nucl., Pisa, Italy, 9-130.

Gowing, J.W., Konukcu, F., Rose, D.A., 2006. Evaporative flux from a shallow water table:

12 the influence of a vapour-liquid phase transition. J. Hydrol.,321, 77-89.

Haverkamp, R., Leij, F.J., Fuentes, C., Sciortino, A., Ross, P.J., 2005. Soil water retention: I.

Introduction of a shape index. Soil Sci. Soc. Am. J. 69, 1881-1890, doi:

1810.2136/sssaj2004.0225.

Keeling CD., 1961. The concentration and isotopic abundances of carbon dioxide in rural and marine air. Geochim. Cosmochim. Acta 24 : 277-298.

Majoube, M.A., 1971. Fractionnement en oxygène-18 et en deutérium entre l'eau et sa vapeur, J. Chem. Phys. 68, 1423-1436.

Merlivat, L., 1978. Molecular diffusivity of $\mathrm{H}_{2}{ }^{16} \mathrm{O}, \mathrm{HD}^{16} \mathrm{O}$ and $\mathrm{H}_{2}{ }^{18} \mathrm{O}$ in gases. J. Chem. Physics 69, 2864-2871.

Saravanapavan, T., Salvucci, G.D, 2000. Analysis of rate-limiting processes in soil evaporation with implications for soil resistance models. Adv. in Water Resour. 23, 493-502. Schneider, K., Ippisch, O., Roth, K., 2006. Novel evaporation experiment to determine soil hydraulic properties. Hydrol. Earth Syst. Sci. 10, 810-827. 
Paper published in Journal of Hydrology, 369, 17-29, 2009, doi:10.1016/j.jhydrol.2009.01.038

1 Van Genuchten, M. T., 1980. A closed-form equation for predicting the hydraulic

2 conductivity of unsaturated soils. Soil Sci. Soc. Am. J. 44, 892-898.

3 Yakir, D., da Silveira Lobo Sternberg, L., 2000. The use of stable isotopes to study ecosystem

4 gas exchange. Oecologia 123, 297-311.

5 
1 List of figures

2 Figure 1 Top: Measured evaporation fluxes $\left(10^{-6} \mathrm{~kg} \mathrm{~m}^{-2} \mathrm{~s}^{-1}\right)$ (light line) versus calculated ones

3 with a constant value of the aerodynamic resistance (black line) for column 1. Bottom:

4 Optimized constant aerodynamic resistance (black horizontal line) versus aerodynamic

5 resistance recalculated using the measured evaporation fluxes and the calculated soil surface

6 specific humidity (light line) for column 1 . The prescribed relative humidity $h_{a}$ is also

7 provided (see Table 3).

8 Figure 2 Comparison of the calculated evaporation fluxes after optimization of the parameters

9 (black line) and the observed values (light line) for columns 1 (top) and 5 (bottom).

Figure 3 Comparison of the observed and calculated soil water content profiles when the six

11 soil columns were dismantled. Black solid lines correspond to model results using an

12 evaporation flux calculated by Eq. (4) and dashed lines are the model results using the measured evaporation flux as boundary condition. For column 4 (bottom middle graph), the model diverged before the end of the simulation when the measured evaporation was used.

Data are only shown for the first $25 \mathrm{~cm}$ depth layer.

Figure 4 Sensitivity of the simulated soil oxygen 18 ratio to the dispersivity length for columns 5 (53 DoD drying), 2 (163 DoD) and 1 (338 DoD). Data are only shown for the first 25 cm depth layer. DoD stands for Day of Drying.

Figure 5 Comparison of the observed and calculated oxygen 18 concentration profiles when the various soil columns were dismantled. Black solid lines correspond to model results using an evaporation flux calculated by Eq. (4) and dashed light lines to model results using the measured evaporation flux as boundary condition. For column 4 (bottom middle graph), the model diverged before the end of the simulation when the measured evaporation was used. Data are only shown for the first $25 \mathrm{~cm}$ depth layer. 
1 Figure 6 Profiles of simulated oxygen 18 fluxes at the end of the drying period of columns 5

2 (53 DoD, DoY 154) (left), 2 (163 DoD, DoY 264) (middle) and 1 (338 DoD, DoY 439)

3 (right). The graphs provide the total (full line), liquid (dotted line) and vapor (dashed line)

4 fluxes profiles within the first $25 \mathrm{~cm}$ depth. DoD stands for Day of Drying. The horizontal

5 lines shows the depth of the peak of oxygen 18 isotopic composition .

$6 \quad$ Figure 7 Left: Ratio of diffusive over total liquid oxygen 18 fluxes for columns 5 (53 DoD)

7 (full line), 2 (163 DoD) (dotted line) and 1 (338 DoD) (dashed line). Right: Ratio of the

8 diffusive over total vapor oxygen 18 fluxes for columns 2 (163 DoD) (dotted line) and 1 (338

9 DoD) (dashed line). Data are only shown for the first $25 \mathrm{~cm}$ (left) and 10cm (right) soil layers.

10 DoD stands for Day of Drying. Horizontal lines show the depth of the peak oxygen 18 content 11 for each profile.

Figure 8 Top: Time evolution of the calculated kinetic fractionation factor (using Eqs. (9) and (14) which provide the same value) for the columns 6 (dotted line), 5 (dashed light line), 3 (full line). The horizontal lines are the literature values of Merlivat (1978) corresponding to $\alpha_{K}=14.0,18.9$ and 28.5 (per mil). Bottom: Soil surface relative humidity for the same columns.

Figure 9 Time evolution of the calculated kinetic fractionation factor using Eqs. (9) (full line) and (14) (dotted line) for the columns 2 (top) and 1 (bottom). Black lines correspond to the optimum value of the dispersivity length $(0.046 \mathrm{~m}$ for column 2 and $0.0055 \mathrm{~m}$ for column 1 see Table 4) and light lines are for $\Lambda=0.01 \mathrm{~m}$. The horizontal lines are the literature values of Merlivat (1978) corresponding to $\alpha_{K}=14.0,18.9$ and 28.5 (per mil). 